\title{
On the Expressivity of ASK Queries in SPARQL
}

\author{
Xiaowang Zhang, ${ }^{1,4}$ Jan Van den Bussche, ${ }^{2}$ Kewen Wang, ${ }^{3}$ \\ Heng Zhang, ${ }^{1}$ Xuanxing Yang, ${ }^{1,4}$ Zhiyong Feng ${ }^{1}$ \\ ${ }^{1}$ College of Intelligence and Computing, Tianjin University, Tianjin, China \\ ${ }^{2}$ Faculty of Sciences, Hasselt University, Hasselt, Belgium \\ ${ }^{3}$ School of Information and Communication Technology, Griffith University, Brisbane, Australia \\ ${ }^{4}$ Tianjin Key Laboratory of Cognitive Computing and Application, Tianjin, China
}

\begin{abstract}
As a major query type in SPARQL, ASK queries are boolean queries and have found applications in several domains such as semantic SPARQL optimization. This paper is a first systematic study of the relative expressive power of various fragments of ASK queries in SPARQL. Among many new results, a surprising one is that the operator UNION is redundant for ASK queries. The results in this paper as a whole paint a rich picture for the expressivity of fragments of ASK queries with the four basic operators of SPARQL 1.0 possibly together with a negation. The work in this paper provides a guideline for future SPARQL query optimization and implementation.
\end{abstract}

\section{Introduction}

The Resource Description Framework (RDF) (Cyganiak, Wood, and Lanthaler 2014), a popular data model for information on the Web, represents information in the form of directed labelled graphs called RDF graphs (e.g., YAGO (Hoffart et al. 2013)), as a currently popular graph database (Angles and Gutiérrez 2008b). The standard query language for RDF data is SPARQL (Prud'hommeaux and Seaborne 2008) with its latest version SPARQL 1.1 (Harris and Seaborne 2013) by extending essential features such as negations, subqueries, regular expressions and aggregation. A SPARQL query is usually expressed in terms of certain algebraic expressions called patterns.

As a major query type in SPARQL (Prud'hommeaux and Seaborne 2008), ASK queries are boolean queries (Gottlob, Leone, and Scarcello 2001) while a SELECT query extracts the set of all result mappings. The importance of boolean queries is well-known in databases (Abiteboul, Hull, and Vianu 1995). In fact, ASK queries have also been applied in semantic SPARQL optimisation (Schmidt, Meier, and Lausen 2010), representing nested queries (Angles and Gutiérrez 2010) and RDF data access from mobile devices with context-aware policies (Costabello, Villata, and Gandon 2012). The expressivity, as an important fundamental property for query optimization and implementation, is a recurring topic in the area of query languages (Angles and Gutiérrez 2008b; Chandra and Harel 1980; Wood 2012).

Copyright (C) 2020, Association for the Advancement of Artificial Intelligence (www.aaai.org). All rights reserved.
The expressive power of SPARQL has been analyzed in its relationship to the relational algebra (Cyganiak 2005), SQL (Chebotko, Lu, and Fotouhi 2009), Datalog (Angles and Gutiérrez 2008a; Polleres 2007), OWL (Gottlob and Pieris 2015), and modal logics (Guido 2015). Also the relationship between expressivity, complexity and optimization of query evaluation has been studied (Pérez, Arenas, and Gutiérrez 2009; Arenas and Pérez 2011; Schmidt, Meier, and Lausen 2010; Letelier et al. 2013; Chekol 2016; Nikolaou and Koubarakis 2016). The internal expressivity of SPARQL has been investigated including regular expressions and property paths (Kostylev et al. 2015), assignment and aggregation (Kaminski, Kostylev, and Grau 2016; 2017), well-designed patterns (Pichler and Skritek 2014; Kaminski and Kostylev 2016), negation and non-monotone operators (Angles and Gutiérrez 2016; Kontchakov and Kostylev 2016), CONSTRUCT queries (Kostylev, Reutter, and Ugarte 2015), operator primitivity (Zhang and den Bussche 2014), navigational power (Zhang and den Bussche 2015), and pattern satisfiability problem (Zhang, den Bussche, and Picalausa 2016; 2016).

However, the expressivity of ASK queries has not been explicitly investigated yet, while some results on SELECT queries and query satisfiability carry forward to ASK queries. Informally, the problem of expressivity of ASK queries is to study the role of an operator in expressing ASK queries. Two SELECT queries are equivalent if they have the same answers for each RDF graph; two queries are equivalent for satisfiability if they have the same satisfiability. Thus, the following implications for the expressivity of ASK queries hold but not the vice versa in general: Expressible for SELECT Query $\Rightarrow$ Expressible for ASK queries $\Rightarrow$ Expressible for query satisfiability.

It implies that when an operator is not expressible for SELECT queries, it could be expressible for ASK queries. For instance, it is known that a SELECT query expressed by $\operatorname{AND}(\mathcal{A})$, MINUS $(\mathcal{M})$ and $\operatorname{UNION}(\mathcal{U})$ cannot be equivalently rewritten into a SELECT query expressed by only AND and MINUS (Zhang et al. 2018). It is interesting to know whether this will hold for ASK queries.

In this paper, we conduct a systematic study on the expressivity of ASK queries in SPARQL. Also, we show some 
results on expressivity that are not reported in the literature while they hold for both SELECT queries and ASK queries. Our major contributions are briefly summarized as follows and specific results are depicted in Figure 1, Figure 2 and Figure 3, respectively. In these figures, a fragment of SPARQL is represented by a sequence of the operators. For instance, $\mathcal{A D O} \mathcal{O}_{F}$ denotes the fragment of ASK queries formed by three operators AND, DIFF and $\mathrm{OPT}_{F}$. All fragments in the same rectangle box are equivalent to each other. If there is an arrow from a box $B_{1}$ to another box $B_{2}$, then every fragment in $B_{1}$ is expressible in a fragment in $B_{2}$.

- We investigate the expressivity of 16 fragments of ASK queries with four basic SPARQL 1.0 operators together with DIFF (see Figure 1).

Figure 1: Expressivity of ASK queries with DIFF

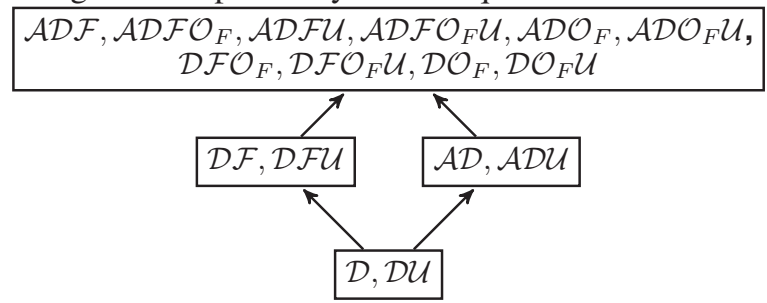

- We investigate the expressivity of 16 fragments of ASK queries with four basic SPARQL 1.0 operators together with MINUS (see Figure 2).

Figure 2: Expressivity of ASK queries with MINUS

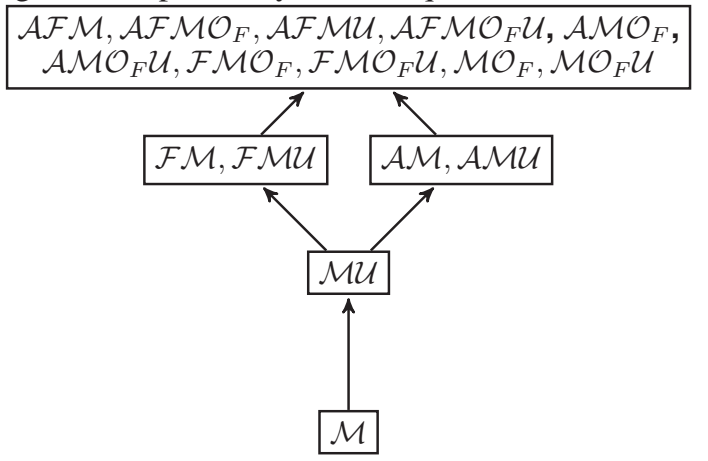

- We investigate the expressivity of 16 fragments of ASK queries with all four basic operators of SPARQL 1.0 (see Figure 3). These results paint a complete picture for the expressivity of the 9 classes (see Figure 3).

This paper is further organised as follows. In the next section, we recall syntax and semantics of SPARQL patterns and ASK queries. Section 3 introduces ASK queries and ASK-expressivity. Section 4 discusses the expressivity of ASK queries with negations and Section 5 discusses the expressivity of ASK queries in SPARQL 1.0. Section 6 concludes our works.
Figure 3: Expressivity of ASK queries in SPARQL 1.0

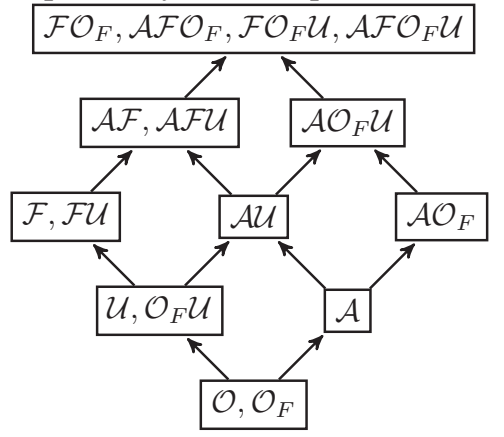

\section{SPARQL: Syntax and Semantics}

In this section, we briefly recall some definitions and notations for the core SPARQL formalization in (Pérez, Arenas, and Gutiérrez 2009; Kontchakov and Kostylev 2016).

\section{RDF graphs}

Let $U$ be the universe of RDF terms consisting of IRIs (i.e., $I$ ) and literals (i.e., $L$ ). An RDF graph (for short, graph) is modeled as a finite set of RDF triples $(s, p, o) \in U \times I \times U$. Following related work (Kontchakov and Kostylev 2016), the distinction between IRIs and literals, on the one hand, and blank nodes and RDF terms on the other hand, does not bring up fundamentally new problems about expressive power. Hence our model of RDF graph is adequate for the purpose of our investigation.

Besides the set $U$ of constants, we also assume that $V$ is an infinite set of variables, which is disjoint from $U$. A variable usually starts with a question mark "?" to distinguish it from a constant. We use $u, v, w$ to denote elements that are either literals or variables; $? x, ? y, ? z$ to denote variables; $a$, $b, c$ to denote constants.

\section{Syntax of SPARQL}

In SPARQL, a query is defined in terms of patterns. A triple $(u, v, w)$ from $(U \cup V) \times(U \cup V) \times(U \cup V)$ is called a triple pattern. A graph pattern (for short, pattern) $P$ is inductively defined as follows:

$$
\begin{array}{r}
P:=(u, v, w) \mid P_{1} \text { AND } P_{2}\left|P_{1} \operatorname{OPT}_{F} P_{2}\right| \\
P_{1} \operatorname{DIFF}_{F} P_{2} \mid P_{1} \text { UNION } P_{2} \mid P_{1} \text { MINUS } P_{2} \mid \\
P_{1} \text { FILTER } F \mid \operatorname{SELECT}_{S}\left(P_{1}\right) .
\end{array}
$$

A filter condition $F$ (or just filter) is a formula constructed from (filter) atoms of the form bnd $(? x), ? x=? y$, and $? x=$ $c$, using logical connectives $\wedge, \vee$, and $\neg$.

For a pattern $P, \operatorname{var}(P)$ and $\operatorname{con}(P)$ denote the set of all variables and the set of all constants in $P$ respectively.

\section{Semantics of SPARQL}

Mappings The semantics of patterns is defined in terms of sets of so-called solution mappings. A solution mapping (simply, mapping) is a total function $\mu: S \rightarrow U$ on a finite set $S$ of variables. The domain $S$ of $\mu$ is denoted 
$\operatorname{dom}(\mu)$. Two mappings $\mu_{1}$ and $\mu_{2}$ are compatible, denoted by $\mu_{1} \sim \mu_{2}$, if they agree on the intersection of their domains. The empty mapping $\left(\mu_{\emptyset}\right)$, whose domain is empty, is always compatible with any mapping.

Given a mapping $\mu$ and a filter $F$, the evaluation of $F$ on $\mu$, denoted by $F^{\mu}$, is defined in terms of a two-valued logic. That is, $F^{\mu}=$ true if $F$ is satisfied by $\mu$ and otherwise $F^{\mu}=$ false (Kontchakov and Kostylev 2016). The standard semantics of SPARQL is three-valued semantics (Pérez, Arenas, and Gutiérrez 2009; Prud'hommeaux and Seaborne 2008). Fortunately, the three-valued semantics of SPARQL can be simulated by the two-valued semantics of SPARQL if FILTER is allowed (Zhang, Meng, and Zou 2018).

SPARQL Algebra Let $\Omega, \Omega_{1}$, and $\Omega_{2}$ be three sets of mappings and $S$ be a set of variables. The SPARQL algebra for sets of mappings is composed of the operations of projection, selection, join, difference, left-join, union, and minus, respectively, defined as follows:

- $\pi_{S}(\Omega)=\left\{\left.\mu\right|_{S \cap \operatorname{dom}(\mu)} \mid \mu \in \Omega\right\}$;

- $\sigma_{F}(\Omega)=\left\{\mu \in \Omega \mid F^{\mu}=\right.$ true $\}$;

- $\Omega_{1} \bowtie \Omega_{2}=\left\{\mu_{1} \cup \mu_{2} \mid \mu_{1} \in \Omega_{1}, \mu_{2} \in \Omega_{2}, \mu_{1} \sim \mu_{2}\right\}$;

$\bullet$

$$
\begin{aligned}
\Omega_{1} \backslash_{F} \Omega_{2}=\left\{\mu_{1} \in\right. & \Omega_{1} \mid \forall \mu_{2} \in \Omega_{2}, \\
& \left.\left(\mu_{1} \not \mu_{2}\right) \vee\left(F^{\mu_{1} \cup \mu_{2}} \neq \text { true }\right)\right\} ;
\end{aligned}
$$

- $\Omega_{1} \cup \Omega_{2}=\left\{\mu \mid \mu \in \Omega_{1} \vee \mu \in \Omega_{2}\right\}$;

$\bullet$

$$
\begin{aligned}
& \Omega_{1} \ominus \Omega_{2}=\left\{\mu_{1} \in \Omega_{1} \mid \forall \mu_{2} \in \Omega_{2},\right. \\
& \left.\left(\mu_{1} \not \mu_{2}\right) \vee\left(\operatorname{dom}\left(\mu_{1}\right) \cap \operatorname{dom}\left(\mu_{2}\right)=\emptyset\right)\right\}
\end{aligned}
$$

- $\Omega_{1} \bowtie_{F} \Omega_{2}=\sigma_{F}\left(\Omega_{1} \bowtie \Omega_{2}\right) \cup\left(\Omega_{1} \backslash_{F} \Omega_{2}\right)$.

Note that we use $\backslash$ and $\triangle \bowtie$ to denote $\backslash_{T}$ and $\triangle_{T}$ respectively. That is,

$$
\begin{gathered}
\Omega_{1} \backslash \Omega_{2}=\left\{\mu_{1} \in \Omega_{1} \mid \forall \mu_{2} \in \Omega_{2}, \mu_{1} \nsim \mu_{2}\right\} . \\
\Omega_{1} \bowtie \Omega_{2}=\left(\Omega_{1} \bowtie \Omega_{2}\right) \cup\left(\Omega_{1} \backslash \Omega_{2}\right) .
\end{gathered}
$$

Clearly, the following equations hold:

$$
\begin{gathered}
\Omega_{1} \backslash{ }_{F} \Omega_{2}=\Omega_{1} \backslash \sigma_{F}\left(\Omega_{1} \bowtie \Omega_{2}\right) . \\
\Omega_{1} \bowtie_{F} \Omega_{2}=\sigma_{F}\left(\Omega_{1} \bowtie \Omega_{2}\right) \cup\left(\Omega_{1} \backslash \sigma_{F}\left(\Omega_{1} \bowtie \Omega_{2}\right)\right) .
\end{gathered}
$$

Semantics of SPARQL Given a graph $G$ and a pattern $P$, the semantics $\llbracket P \rrbracket_{G}$ of $P$ on $G$ is defined by a set of mappings as follows:

$$
\begin{aligned}
& \llbracket(u, v, w) \rrbracket_{G}=\{\mu:\{u, v, w\} \cap V \rightarrow U \mid \\
&(\mu(u), \mu(v), \mu(w)) \in G\} .
\end{aligned}
$$

Here $\mu(c)=c$ for any $c \in U$.

- $\llbracket P_{1}$ UNION $P_{2} \rrbracket_{G}=\llbracket P_{1} \rrbracket_{G} \cup \llbracket P_{2} \rrbracket_{G}$;

- $\llbracket P_{1}$ AND $P_{2} \rrbracket_{G}=\llbracket P_{1} \rrbracket_{G} \bowtie \llbracket P_{2} \rrbracket_{G}$;

- $\llbracket P_{1} \quad \mathrm{DIFF}_{F} \quad P_{2} \rrbracket_{G}=\llbracket P_{1} \rrbracket_{G} \backslash_{F} \llbracket P_{2} \rrbracket_{G}$;
- $\llbracket P_{1} \quad$ MINUS $P_{2} \rrbracket_{G}=\llbracket P_{1} \rrbracket_{G} \ominus \llbracket P_{2} \rrbracket_{G}$;

- $\llbracket P_{1}$ FILTER $F \rrbracket_{G}=\sigma_{F}\left(\llbracket P_{1} \rrbracket_{G}\right)$;

- $\llbracket P_{1} \mathrm{OPT}_{F} P_{2} \rrbracket_{G}=\llbracket P_{1} \rrbracket_{G} \searrow_{F} \llbracket P_{2} \rrbracket_{G} ;$

- $\llbracket \operatorname{SELECT}_{S}\left(P_{1}\right) \rrbracket_{G}=\pi_{S}\left(\llbracket P_{1} \rrbracket_{G}\right)$.

Clearly, the semantics of $P_{1}$ DIFF $P_{2}$ and $P_{1}$ OPT $P_{2}$ on $G$ are equivalently defined as follows:

$$
\begin{array}{lll}
\llbracket P_{1} & \text { DIFF } & P_{2} \rrbracket_{G}=\llbracket P_{1} \rrbracket_{G} \backslash \llbracket P_{2} \rrbracket_{G} . \\
\llbracket P_{1} & \text { OPT } & P_{2} \rrbracket_{G}=\llbracket P_{1} \rrbracket_{G} \bowtie \llbracket P_{2} \rrbracket_{G} .
\end{array}
$$

Standard Equivalence: Two patterns $P$ and $P^{\prime}$ are equivalent, denoted $P \equiv P^{\prime}$, if $\llbracket P \rrbracket_{G}=\llbracket P^{\prime} \rrbracket_{G}$ for any graph $G$.

By the definition, we have the following lemma.

Lemma 1. Let $P_{1}$ and $P_{2}$ be two patterns and $F$ a filter. For any graph $G$, the following two equations hold:

-

$P_{1} \operatorname{DIFF}_{F} P_{2}=P_{1} \operatorname{DIFF}\left(\left(P_{1}\right.\right.$ AND $\left.P_{2}\right)$ FILTER $\left.F\right)$

-

$$
\begin{array}{r}
P_{1} \mathrm{OPT}_{F} P_{2}=\left(\left(P_{1} \text { AND } P_{2}\right) \text { FILTER } F\right) \text { UNION } \\
\left(P_{1} \operatorname{DIFF}\left(\left(P_{1} \text { AND } P_{2}\right) \text { FILTER } F\right) .\right.
\end{array}
$$

By Lemma 1, $\mathrm{DIFF}_{F}$ and $\mathrm{OPT}_{F}$ are not necessary if AND, FILTER, UNION and DIFF are allowed.

\section{ASK Queries and ASK-Expressivity}

In this section, we define the equivalence of ASK queries and then formalise the expressivity problem of ASK queries.

\section{ASK Query and Expressivity}

An $A S K$ query $Q$ is of the form $\operatorname{ASK}(P)$, where $P$ is a pattern. When there is no confusion, an ASK query is just called a query.

Semantically, given a graph $G$, the evaluation of $\operatorname{ASK}(P)^{G}$ is true if $\llbracket P \rrbracket_{G} \neq \emptyset$; and false otherwise.

Definition 2. Two ASK queries $Q_{1}$ and $Q_{2}$ are equivalent, denoted $Q_{1} \equiv_{\mathrm{ASK}} Q_{2}$, if for every graph $G, Q_{1}^{G}=$ true iff $Q_{2}^{G}=$ true. If $Q_{i}=\operatorname{ASK}\left(P_{i}\right)$ for $i=1,2$ and $Q_{1} \equiv \mathrm{ASK}$ $Q_{2}$, then we say that $P_{1}$ and $P_{2}$ are $A S K$-equivalent, denoted $P_{1} \equiv$ ASK $P_{2}$.

We say that $Q$ is $A S K$-expressible, or just expressible, in a fragment $\mathcal{W}$ if $Q \equiv_{\mathrm{ASK}} Q^{\prime}$ for some query $Q^{\prime}$ in $\mathcal{W}$.

A fragment $\mathcal{W}_{1}$ is ASK-expressible, or just expressible, in another fragment $\mathcal{W}_{2}$, denoted by $\mathcal{W}_{1} \preceq \mathcal{W}_{2}$, if every ASK query in $\mathcal{W}_{1}$ is expressible in $\mathcal{W}_{2}$. Otherwise, $\mathcal{W}_{1} \npreceq \mathcal{W}_{2}$. By $\mathcal{W}_{1} \npreceq \mathcal{W}_{2}$, we mean $\mathcal{W}_{1} \preceq \mathcal{W}_{2}$ and $\mathcal{W}_{2} \npreceq \mathcal{W}_{1}$.

An ASK query $Q$ is monotone if for each pair of graphs $G_{1}$ and $G_{2}$ with $G_{1} \subseteq G_{2}, Q^{G_{1}}=$ true implies $Q^{G_{2}}=$ true; and non-monotone otherwise. A fragment $\mathcal{W}$ of ASK queries is monotone if all ASK queries in $\mathcal{W}$ are monotone; and non-monotone otherwise. An operator $\mathcal{X}$ is monotone if the fragment consisting of only $\mathcal{X}$ is monotone; and non-monotone otherwise. DIFF $F$ and MINUS are nonmonotone, while AND, FILTER, $\mathrm{OPT}_{F}$, and UNION are 
monotone under ASK queries. Note that $\mathrm{OPT}_{F}$ is monotone in ASK queries while it is non-monotone in SELECT queries (Kontchakov and Kostylev 2016).

As pointed out in (Zhang, den Bussche, and Picalausa 2016), SELECT can be removed by a renaming of variables. Thus, we assume that ASK queries are SELECT-free.

\section{Expressivity of ASK Queries with Negations}

In this section, we discuss the expressivity of ASK queries with negations $\left(\right.$ DIFF $_{F}$, DIFF, and MINUS).

\section{Expressivity of ASK queries with DIFF $_{F}$}

It is easy to see that there are 16 fragments of SPARQL that are extensions of the fragment $\mathcal{A F O U}$ by adding $\mathrm{DIFF}_{F}$. In this subsection, we investigate the expressive power of ASK queries in these fragments. A major result is that AND cannot be expressed by $\mathrm{DIFF}_{F}$.

We first show that ASK queries with FILTER is expressible in ASK queries with $\operatorname{DIFF}_{F}$.

Proposition 3. Let $P$ be a pattern and $F$ be a filter. Then

$P$ FILTER $F \equiv P \operatorname{DiFF}\left(P \operatorname{DiFF}_{F}(? x, ? y, ? z)\right)$, where $? x, ? y, ? z$ are fresh variables.

By definition, it follows that $\mathrm{OPT}_{F}$ is expressible by AND, DIFF $_{F}$ and UNION while FILTER is expressible by only $\operatorname{DIFF}_{F}$ and UNION.

Proposition 4. The following hold.

- $\mathcal{A D}_{F} \mathcal{F} \mathcal{O}_{F} \mathcal{U} \preceq \mathcal{A D} \mathcal{D}_{F} \mathcal{U}$;

- $\mathcal{D}_{F} \mathcal{F U} \preceq \mathcal{D}_{F} \mathcal{U}$.

The next result shows that UNION of ASK queries in $\mathcal{A D}_{F} \mathcal{U}$ and $\mathcal{D}_{F} \mathcal{U}$ is essentially redundant in terms of the expressivity of ASK queries.

A pattern $P$ is in UNION normal form (UNF) if $P$ is of the form

$$
P_{1} \text { UNION } \cdots \text { UNION } P_{m}
$$

where $P_{i}$ is a UNION-free pattern $(i=1, \ldots, m)$.

Proposition 5. If $P$ is a UNF pattern in Equation (1), then

$$
\begin{aligned}
P \equiv \text { ASK } & (? x, ? y, ? z) \text { DIFF } \\
& \left(\left(\left(? x^{\prime}, ? y^{\prime}, ? z^{\prime}\right) \operatorname{DIFF} P_{1}\right) \cdots \operatorname{DIFF} P_{m}\right)
\end{aligned}
$$

where $? x, ? y, ? z, ? x^{\prime}, ? y^{\prime}, ? z^{\prime}$ are fresh variables that do not appear in $P$.

In the above proof of Proposition 5, the idea is essentially based on the following statement.

Proposition 6. Let $Q, P_{1}, \ldots, P_{m}$ are patterns. Then

$$
\begin{aligned}
& Q \operatorname{DIFF}\left(P_{1} \mathrm{UNION} \cdots \mathrm{UNION} P_{m-1} \mathrm{UNION} P_{m}\right) \\
& \equiv \mathrm{ASK}\left(\left(Q \operatorname{DIFF} P_{1}\right) \cdots \operatorname{DIFF} P_{m-1}\right) \operatorname{DIFF} P_{m} .
\end{aligned}
$$

Since each pattern in $\mathcal{A D U}$ is equivalent to a UNF pattern (Zhang et al. 2018), by Propositions 5 and 6, we have the following corollary.

Corollary 7. The following hold.

- $\mathcal{D}_{F} \mathcal{U} \preceq \mathcal{D}_{F}$;
- $\mathcal{A D} \mathcal{D}_{F} \mathcal{U} \preceq \mathcal{A} \mathcal{D}_{F}$.

It is interesting to see whether $\mathcal{A}$ is expressible in $\mathcal{D}_{F}$. The answer is negative.

Proposition 8. $\mathcal{A} \npreceq \mathcal{D}_{F}$.

Now, we can present our main theorem in this subsection.

Theorem 9. The following statements hold for ASK queries in SPARQL (shown in Figure 4):

1. All fragments in $\mathbf{W}_{1}=\left\{\mathcal{D}_{F}, \mathcal{D}_{F} \mathcal{F}, \mathcal{D}_{F} \mathcal{F U}, \mathcal{D}_{F} \mathcal{U}\right\}$ are equivalent to each other.

2. All fragments in $\mathbf{W}_{2}=\left\{\mathcal{A D}_{F}, \mathcal{A D}_{F} \mathcal{F}, \mathcal{A D}_{F} \mathcal{F} \mathcal{O}_{F}\right.$, $\mathcal{A D}_{F} \mathcal{F} \mathcal{O}_{F} \mathcal{U}, \mathcal{A D}_{F} \mathcal{F} \mathcal{U}, \mathcal{A D}_{F} \mathcal{O}_{F}, \mathcal{A D} \mathcal{D}_{F} \mathcal{O}_{F} \mathcal{U}, \mathcal{A D _ { F }} \mathcal{U}$, $\left.\mathcal{D}_{F} \mathcal{F} \mathcal{O}_{F}, \mathcal{D}_{F} \mathcal{F} \mathcal{O}_{F} \mathcal{U}, \mathcal{D}_{F} \mathcal{O}_{F}, \mathcal{D}_{F} \mathcal{O}_{F} \mathcal{U}\right\}$ are equivalent to each other.

3. If $\mathcal{W}_{1} \in \mathbf{W}_{1}$ and $\mathcal{W}_{2} \in \mathbf{W}_{2}$, then $\mathcal{W}_{1} \supsetneqq \mathcal{W}_{2}$.

Figure 4: Expressivity of ASK queries with $\mathrm{DIFF}_{F}$ $\mathcal{A D}_{F}, \mathcal{A D}_{F} \mathcal{F}, \mathcal{A D}_{F} \mathcal{F} \mathcal{O}_{F}, \mathcal{A D _ { F }} \mathcal{F} \mathcal{O}_{F} \mathcal{U}, \mathcal{A D}_{F} \mathcal{F U}, \mathcal{A D}_{F} \mathcal{O}_{F}$
$\mathcal{A D}_{F} \mathcal{O}_{F} \mathcal{U}, \mathcal{A} \mathcal{D}_{F} \mathcal{U}, \mathcal{D}_{F} \mathcal{F} \mathcal{O}_{F}, \mathcal{D}_{F} \mathcal{F} \mathcal{O}_{F} \mathcal{U}, \mathcal{D}_{F} \mathcal{O}_{F}, \mathcal{D}_{F} \mathcal{O}_{F} \mathcal{U}$

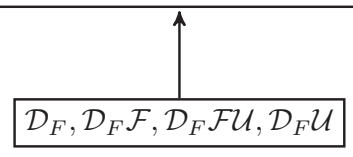

\section{Expressivity of ASK queries with either DIFF or MINUS}

In this subsection, we consider the 32 fragments of $\mathcal{A F} \mathcal{F}_{F} \mathcal{U}$ with either DIFF or MINUS.

We show that DIFF $_{F}$ is expressible in $\mathcal{M O}_{F}$ and $\mathcal{D O} \mathcal{O}_{F}$.

Proposition 10. Let $P_{1}$ and $P_{2}$ be two patterns in $\mathcal{A D}_{F}$. Then $P_{1}$ DIFF $_{F} \quad P_{2} \equiv P$ MINUS $(? x, ? y, ? z)$ where $? x, ? y, ? z$ are fresh variables and $P=\left(P_{1} \mathrm{OPT}_{F}\right.$ $\left.\left(P_{2} \operatorname{OPT}(? x, ? y, ? z)\right)\right)$.

It is shown that AND is expressible in $\mathcal{M O}_{F}$ (Zhang and den Bussche 2014). By Proposition $10, \mathcal{A D}_{F}$ is expressible in $\mathcal{M O}_{F}$.

Now we wonder to know whether $\mathcal{A D}_{F}$ is expressible in $\mathcal{D} \mathcal{O}_{F}$. The answer is positive.

Let $P$ be a pattern and $F$ a filter. Filter $(P, F)$ denotes the pattern $P$ DIFF $P_{\neg F}$, where $P_{\neg F}$ is defined as

$$
\begin{array}{r}
\left(P \mathrm { OPT } _ { F \wedge ? x _ { 1 } = ? x _ { 2 } } \left(\left(? x_{1}, ? y_{1}, ? z_{1}\right)\right.\right. \\
\left.\left.\operatorname{OPT}\left(? x_{2}, ? y_{2}, ? z_{2}\right)\right)\right) \operatorname{DIFF} Q,
\end{array}
$$

$$
\begin{array}{r}
Q=\left(\left(\left(? x_{1}, ? y, ? z\right) \mathrm{OPT}_{? x_{1} \neq ? x_{2}}\left(? x_{2}, ? u_{1}, ? v_{1}\right)\right)\right. \\
\left.\mathrm{OPT}_{? x_{1} \neq ? x_{2}}\left(? u_{2}, ? x_{2}, ? v_{2}\right)\right) \\
\mathrm{OPT}_{? x_{1} \neq ? x_{2}}\left(? u_{3}, ? v_{3}, ? x_{2}\right)
\end{array}
$$

where $? y, ? z, ? x_{1}, ? x_{2}, ? x_{3}, ? y_{1}, ? y_{2}, ? z_{1}, ? z_{2}, ? u_{i}, ? v_{i}(i=$ $1,2,3)$ are fresh variables.

Proposition 11. Let $P$ be a pattern and $F$ be a filter. For any graph $G$, if $G$ contains at least two constants then:

$$
\llbracket P \text { FILTER } F \rrbracket_{G}=\llbracket F i l t e r(P, F) \rrbracket_{G} .
$$


However, Proposition 11 does not hold if $G$ consists of only one constant of the form $\{(c, c, c)\}$ (that is, $G$ is a singleton complete graph). In this case, $\llbracket P$ DIFF $P_{\neg F} \rrbracket_{G} \neq \emptyset$ since $\llbracket P_{F} \rrbracket_{G}=\emptyset$ no matter what $F$ is.

So, we consider all patterns in $\mathcal{A D} \mathcal{F} \mathcal{O}_{F} \mathcal{U}$ but graphs containing only one constant.

For the moment we consider the fragment $\mathcal{A D} \mathcal{F}_{0}$ where all triple patterns are constant-free and all filters are atomic.

Given a pattern $P$ in $\mathcal{A D}_{0}$, its mapping schema, denoted by $\Gamma(P)$, is defined as a set of variables by

- $\Gamma((? u, ? v, ? w))=\{? u, ? v, ? w\}$;

- $\Gamma\left(P_{1}\right.$ AND $\left.P_{2}\right)=\Gamma\left(P_{1}\right) \cap \Gamma\left(P_{2}\right)$;

- $\Gamma\left(P_{1} \operatorname{DIFF} P_{2}\right)=\Gamma\left(P_{1}\right)$;

- $\Gamma\left(P_{1}\right.$ FILTER $\left.F\right)=\Gamma\left(P_{1}\right)$.

A pattern $P$ in $\mathcal{A D} \mathcal{F}_{0}$ is said to be safe if $\operatorname{var}(F) \subseteq \Gamma\left(P_{1}\right)$ for every subpattern $Q$ of the form $P_{1}$ FILTER $F$. We remark that this definition extends the definition of safe pattern in (Pérez, Arenas, and Gutiérrez 2009) for supporting negated patterns.

Firstly, we consider the safe fragment $\mathcal{A D} \mathcal{F}_{0}^{\text {safe }}$ where all patterns are safe and all filters are atomic.

Let $? x_{0}$ be a fixed variable and $P$ be a pattern in $\mathcal{A D} \mathcal{F}_{0}^{\mathrm{safe}}$. We define $\delta(P)$ as an $\mathcal{A D}$-pattern in an inductive way.

- $\delta(? u, ? v, ? w)=\left(? x_{0}, ? x_{0}, ? x_{0}\right)$;

- $\delta\left(P_{1} \operatorname{AND} P_{2}\right)=\delta\left(P_{1}\right) \operatorname{AND} \delta\left(P_{2}\right)$;

- $\delta\left(P_{1} \operatorname{DIFF} P_{2}\right)=\delta\left(P_{1}\right) \operatorname{DIFF} \delta\left(P_{2}\right)$;

- $\delta(P$ FILTER bound $(? y))=\delta(P)$;

- $\delta(P \quad$ FILTER $\neg$ bound $(? y))=\delta(P)$ DIFF $\left(? x_{0}, ? x_{0}, ? x_{0}\right)$

- $\delta(P$ FILTER $? y=? z)=\delta(P)$;

- $\delta(P$ FILTER $? y \neq ? z)=\delta(P) \operatorname{DIFF}\left(? x_{0}, ? x_{0}, ? x_{0}\right)$;

- $\delta(P$ FILTER ? $y=c)=\delta(P) \operatorname{AND}(c, c, c)$;

- $\delta(P$ FILTER $? y \neq c)=\delta(P) \operatorname{DIFF}(c, c, c)$.

The definition of $\delta(P)$ can be extended in $\mathcal{A D} \mathcal{F U}$.

Lemma 12. Let $P$ be a patten in $\mathcal{A D \mathcal { F }} \mathcal{O}_{F} \mathcal{U}$. For any $R D F$ graph $G$ of the form $\{(c, c, c)\}$ with $c \in U$, the following hold:

\section{$\llbracket P \rrbracket_{G}$ is nonempty iff $\llbracket \delta(P) \rrbracket_{G}$ is nonempty.}

By Proposition 11 and Lemma 12, we can show that patterns in $\mathcal{A D} \mathcal{F} \mathcal{O}_{F} \mathcal{U}$ are expressible in $\mathcal{D O} \mathcal{O}_{F}$.

We first prove a proposition.

Given a pattern $P$ in $\mathcal{A D} \mathcal{F} \mathcal{O}_{F} \mathcal{U}$, we define

$$
P^{*}=P^{F} \operatorname{UNION}\left(\delta(P) \text { AND } P_{\top}\right) ;
$$

where $P^{F}$ is a FILTER-free pattern by substituting Filter $\left(P^{\prime}, F\right)$ for all sub-patterns of the form $P^{\prime}$ FILTER $F$ in $P, P_{\top}$ is defined by

$$
\begin{array}{r}
P_{\top}=\left(( ? x _ { 3 } , ? y _ { 3 } , ? z _ { 3 } ) \mathrm { OPT } _ { ? x _ { 1 } = ? x _ { 2 } } \left(\left(? x_{1}, ? y_{1}, ? z_{1}\right)\right.\right. \\
\text { OPT } \left.\left.\left(? x_{2}, ? y_{2}, ? z_{2}\right)\right)\right) \operatorname{DIFF} Q .
\end{array}
$$

Here $Q$ is defined in Equation (4) and $? x_{i}, ? y_{i}, ? z_{i}(i=$ $1,2,3)$ are fresh variables.
Proposition 13. Let $P$ be a pattern in $\mathcal{A D F} \mathcal{O}_{F} \mathcal{U}$ and the pattern $P^{*}$ is defined as above. Then $P \equiv{ }_{\mathrm{ASK}} P^{*}$.

Now, we show that $\mathcal{A D} \mathcal{F} \mathcal{O}_{F} \mathcal{U}$ is expressible in $\mathcal{D} \mathcal{O}_{F}$.

Theorem 14. $\mathcal{A D} \mathcal{F} \mathcal{O}_{F} \mathcal{U} \preceq \mathcal{D O} \mathcal{O}_{F}$.

By Theorems 9, 13 and 14, we conclude the following.

Corollary 15. The following hold.

- $\mathcal{A} \mathcal{F} \mathcal{M O}_{F} \mathcal{U} \preceq \mathcal{M O} \mathcal{O}_{F}$.

- $\mathcal{A D F} \mathcal{O}_{F} \mathcal{U} \preceq \mathcal{D O} \mathcal{O}_{F}$.

Next, we show that $\mathrm{OPT}_{F}$ is necessary to express FILTER when DIFF $_{F}$ is absent. This is due to the fact that equality-constraints of FILTER are not expressible in any equality-free fragment (Casanovas, Dellunde, and Jansana 1996).

Since DIFF can be rewritten by MINUS syntactically in $\mathcal{A D} \mathcal{M U}$, we have the following result.

Proposition 16. $\mathcal{A D} \mathcal{M U} \preceq \mathcal{A M U}$.

In the proof of Proposition 16, in a pattern $P$ in $\mathcal{A D} \mathcal{M U}$, for any subpattern $Q$ of the form $P_{1}$ DIFF $P_{2}$, we can rewrite it into $P_{1}$ MINUS $\left(P_{1}\right.$ AND $\left.P_{2}\right)$.

We will show that FILTER is not expressible in $\mathcal{A D} \mathcal{M U}$. By Proposition 16, we consider only the fragment $\mathcal{A M U}$ instead of $\mathcal{A D} \mathcal{M U}$.

It is in order to introduce the notion of surjective strict homomorphism for graphs.

Definition 17. Let $G$ and $G^{\prime}$ be two graphs and $C$ be a set of constants. A surjective strict $C$-homomorphism from $G$ to $G^{\prime}$ is a surjective function $h: \operatorname{const}(G) \rightarrow \operatorname{const}\left(G^{\prime}\right)$ such that $(s, p, o) \in G$ iff $(h(s), h(p), h(o)) \in G^{\prime}$ for all constants $s, p, o \in \operatorname{const}(G)$, and $h(c)=c$ for all $c \in C$.

Lemma 18. Let $P$ be a pattern in $\mathcal{A M U}$ and $C=\operatorname{con}(P)$. Let $G$ and $G^{\prime}$ be two graphs such that there is a surjective strict $C$-homomorphism h from $G$ onto $G^{\prime}$. Then

1. $\mu \in \llbracket P \rrbracket_{G}$ iff $\mu \circ h \in \llbracket P \rrbracket_{G^{\prime}}$;

2. If $\mu \in \llbracket P \rrbracket_{G^{\prime}}$, then $\mu=\mu^{\prime} \circ h$ for some $\mu^{\prime} \in \llbracket P \rrbracket_{G}$.

Note that $\mathrm{OPT}_{F}$ is needed for expressing FILTER.

By Lemma 18 and Proposition 16, we can show the following theorem.

Theorem 19. $\mathcal{F} \npreceq \mathcal{A D} \mathcal{M U}$.

Proof. Consider the pattern $P=(? x, p, ? y)$ FILTER $? x \neq$ $? y$ with $C=\{p\}$. Take $G=\{(a, p, a),(a, p, b),(b, p, a)$, $(b, p, b)\}$ and $G^{\prime}=\{(a, p, a)\}$. Let $h$ be a function such that

$$
h(a)=h(b)=a \text { and } h(p)=p .
$$

Clearly, $h$ is a surjective strict $C$-homomorphism $h$ from $G$ to $G^{\prime}$. It is not difficult to see that $\llbracket P \rrbracket_{G} \neq \emptyset$. On the other hand, by Lemma 18, $P \rrbracket_{G^{\prime}}=\emptyset$, a contradiction.

By Theorem 19, we have $\mathcal{F} \npreceq \mathcal{A M U}$ and $\mathcal{F} \npreceq \mathcal{A D U}$. We note that $\mathcal{D} \mathcal{M U}$ is expressible in GF (Zhang et al. 2018) and cyclic join queries are not expressible in GF (Flum, Frick, and Grohe 2002; Gottlob, Grädel, and Veith 2002) while cyclic join queries can be expressed by AND in the proof of Proposition 8. Also, we have the following. 
Proposition 20. $\mathcal{A} \npreceq \mathcal{D} \mathcal{M U}$.

By Proposition 20, we have that $\mathcal{A} \npreceq \mathcal{M U}$ and $\mathcal{A} \npreceq \mathcal{D U}$. Next, we would like to know whether ASK queries in a fragment of $\mathcal{A F D U}$ are expressible in the corresponding fragment with UNION. The answer is positive.

To do so, we need an important lemma.

Lemma 21. Let $P$ be a pattern in UNF as Equation (1) and $P^{\prime}$ be the UNION-free pattern

$$
\begin{array}{r}
(? x, ? y, ? z) \operatorname{DIFF}\left(\left(\left(? x^{\prime}, ? y^{\prime}, ? z^{\prime}\right) \operatorname{DIFF}\left(P_{1}\right)\right) \cdots\right. \\
\left.\operatorname{DIFF}\left(P_{m}\right)\right),
\end{array}
$$

where $? x, ? y, ? z, ? x^{\prime}, ? y^{\prime}, ? z^{\prime}$ are fresh variables.

Since each pattern in $\mathcal{A F D U}$ is equivalent to an $\mathcal{A F D}$ pattern in UNF, by Lemma 21, we conclude the following.

Proposition 22. The following hold.

- $\mathcal{A D U} \preceq \mathcal{A D}$;

- $\mathcal{F D U} \preceq \mathcal{F D}$;

- $\mathcal{D U} \preceq \mathcal{D}$.

Finally, we show that ASK queries in a fragment of $\mathcal{A F M U}$ are expressible in the corresponding fragment without UNION.

Proposition 23. $\mathcal{A M U} \preceq \mathcal{A M}$.

Let $P$ be a pattern in $\mathcal{F} \mathcal{M U}$. We use $\operatorname{pos}(P)$ to denote the set of triple patterns in $P$ as follows.

- $\operatorname{pos}((u, v, w))=\{(u, v, w)\}$;

- $\operatorname{pos}\left(P_{1}\right.$ UNION $\left.P_{2}\right)=\operatorname{pos}\left(P_{1}\right) \cup \operatorname{pos}\left(P_{2}\right)$;

- $\operatorname{pos}\left(P_{1}\right.$ MINUS $\left.P_{2}\right)=\operatorname{pos}\left(P_{1}\right)$;

- $\operatorname{pos}\left(P_{1}\right.$ FILTER $\left.F\right)=\operatorname{pos}\left(P_{1}\right)$.

We are ready to show that UNION is expressible by FILTER and MINUS.

Proposition 24. $\mathcal{F} \mathcal{M U} \preceq \mathcal{F} \mathcal{M}$.

Surprisingly, different from DIFF, MINUS cannot express UNION.

Proposition 25. $\mathcal{U} \npreceq \mathcal{M}$.

Proof. (Sketch) Consider the pattern $Q=$ $\operatorname{ASK}(a, a, a)$ UNION $(b, b, b)$ where $a, b \in U$.

Claim 26. There exists no pattern $P$ in $\mathcal{M}$ such that for every RDF graph $G, Q^{G}=$ true iff $\operatorname{ASK}(P)^{G}=$ true.

In order to prove this claim, consider three graphs:

- $G_{1}=\{a, b\} \times\{a, b\} \times\{a, b\} \backslash\{(a, a, a),(b, b, b)\}$.

- $G_{2}=\{a, b\} \times\{a, b\} \times\{a, b\} \backslash\{(a, a, a)\}$.

- $G_{3}=\{a, b\} \times\{a, b\} \times\{a, b\} \backslash\{(b, b, b)\}$.

Note that $Q^{G_{1}}=$ false but $Q^{G_{2}}=$ true and $Q^{G_{3}}=$ true. However, there exists no pattern $P$ in $\mathcal{M}$ such that $P^{G_{1}}=$ false but $P^{G_{2}}=$ true and $P^{G_{3}}=$ true.

Therefore, we can conclude that $\mathcal{U} \npreceq \mathcal{M}$.
Clearly, by Proposition 25, it is immediate to conclude that $\mathcal{M U} \npreceq \mathcal{M}$.

In the proof of $\mathcal{U} \npreceq \mathcal{M}$, we consider patterns with only constants. So we wonder whether a pattern in $\mathcal{U}$ is expressible in $\mathcal{M}$ if each of triple patterns in the given pattern contains a variable. Fortunately, the answer is positive.

Proposition 27. Let $P$ be a pattern in $\mathcal{M U}$. If $\operatorname{pos}(P)$ contains no variable-free triple patterns, then $\operatorname{ASK}(P)$ can be expressed in $\mathcal{M}$.

\section{Expressivity of ASK queries in SPARQL 1.0}

In this section, we discuss the expressivity of ASK queries in $\mathcal{A} \mathcal{F} \mathcal{O}_{F} \mathcal{U}$ (i.e., SPARQL 1.0). We study the expressivity of each operator from FILTER, UNION, $\mathrm{OPT}_{F}$ and AND. So, there are four cases.

The case of FILTER is easy. We show that an ASK query containing FILTER is not expressible in $\mathcal{A O}_{F} \mathcal{U}$. That is, $\mathcal{F} \npreceq \mathcal{A O}_{F} \mathcal{U}$. To see this, we consider pattern $P=(? x, ? y, ? z)$ FILTER $? x \neq ? y$ and graph $G=$ $\{(c, c, c)\}$. Then $\llbracket P \rrbracket_{G}=\emptyset$. We will show that if a pattern $P^{\prime}$ is in $\mathcal{A O}_{F} \mathcal{U}$, then $\llbracket P^{\prime} \rrbracket_{G} \neq \emptyset$. Thus, the pattern $P$ cannot be expressed in $\mathcal{A O}_{F} \mathcal{U}$. It is sufficient to show that the UNION, $\mathrm{OPT}_{F}$ and AND of two triple patterns $(u, v, w)$ and $\left(u^{\prime}, v^{\prime}, w^{\prime}\right)$ is always nonempty.

Case $1 \llbracket(u, v, w)$ UNION $\left(u^{\prime}, v^{\prime}, w^{\prime}\right) \rrbracket_{G}$ contains two mappings $\mu: \operatorname{var}((u, v, w)) \rightarrow\{c\}$ and $\mu^{\prime}$ : $\operatorname{var}\left(\left(u^{\prime}, v^{\prime}, w^{\prime}\right)\right) \rightarrow\{c\}$.

Case $2 \llbracket(u, v, w) \operatorname{AND}\left(u^{\prime}, v^{\prime}, w^{\prime}\right) \rrbracket_{G}$ contains one mapping $\mu: \operatorname{var}((u, v, w)) \cup \operatorname{var}\left(\left(u^{\prime}, v^{\prime}, w^{\prime}\right)\right) \rightarrow\{c\}$.

Case $3 \llbracket(u, v, w) \operatorname{OPT}_{F}\left(u^{\prime}, v^{\prime}, w^{\prime}\right) \rrbracket_{G}$ contains one mapping $\mu$ : $\operatorname{var}((u, v, w)) \cup \operatorname{var}\left(\left(u^{\prime}, v^{\prime}, w^{\prime}\right)\right) \rightarrow\{c\}$.

Given $G=(c, c, c)$ and $P^{\prime}$ in $\mathcal{A O U}$, two mappings in $\llbracket P^{\prime} \rrbracket_{G}$ are always compatible. Therefore, $\llbracket P^{\prime} \rrbracket_{G} \neq \emptyset$ for each $P^{\prime}$ in $\mathcal{A O U}$. This implies $P$ is not expressible in $\mathcal{A O U}$.

In the rest of this section, we investigate the expressivity of the other cases.

\section{Expressivity of ASK queries with UNION}

It is known that UNION is a primitive operator under select queries. It would be interesting to see whether this holds for ASK queries. We will demonstrate that this is not the case for ASK queries. Specifically, when $\mathrm{OPT}_{F}$ is absent, each ASK query with UNION can be expressed by some ASK query with FILTER and AND.

Proposition 28. The following hold.

- $\mathcal{A F U} \preceq \mathcal{A F}$;

- $\mathcal{F U} \preceq \mathcal{F}$.

From Proposition 28, we see that FILTER can be used to express ASK queries with UNION. Moreover, the next proposition shows that FILTER is necessary. We need a lemma before proving the proposition.

Lemma 29. Let $G_{1}=\{(a, a, a)\}$ and $G_{2}=\{(b, b, b)\}$ be two graphs. For every pattern $P$ in $\mathcal{A O}_{F}$, if both $\llbracket P \rrbracket G_{1}$ and $\llbracket P \rrbracket_{G_{2}}$ are nonempty then $\llbracket P \rrbracket_{G_{3}}$ is nonempty for any graph $G_{3}=\{(c, c, c)\}$ with $c \notin \operatorname{con}(P)$. 
This lemma can be shown by a simple induction on the structure of patterns.

Proposition 30. $\mathcal{U} \npreceq \mathcal{A O}_{F}$.

When $\mathrm{OPT}_{F}$ is involved, the conclusion of Proposition 28 still holds.

Proposition 31. $\mathcal{A F} \mathcal{F O}_{F} \mathcal{U} \preceq \mathcal{F} \mathcal{O}_{F}$.

\section{Expressivity of ASK queries with OPT}

In this subsection we show that OPT is necessary in SPARQL 1.0 if UNION is absent. However, UNION is strong enough for expressing OPT. This result can be proven by the (non)-monotonicity of fragment of ASK queries.

We note that both $\mathcal{A F U}$ and $\mathcal{O}_{F} \mathcal{U}$ of ASK queries are monotone. But $\mathcal{A O}_{F}$ is non-monotone. To see this, consider the pattern $P$ as follows:

$$
P=((? x, p, ? y) \text { OPT }(? x, q, ? z)) \text { AND }(? y, r, ? z) .
$$

Let $G_{1}=\{(a, p, b),(b, r, c)\}$ and $G_{2}=G_{1} \cup\{(a, q, b)\}$. Then $\llbracket P \rrbracket_{G_{1}}=\{\{? x \rightarrow a, ? y \rightarrow b, ? z \rightarrow c\}\}$ while $\llbracket P \rrbracket_{G_{2}}=\emptyset$. Thus, $P$ is not monotone. This implies $\mathcal{A O}_{F}$ is non-monotone.

Proposition 32. The following hold.

- $\mathcal{A O}_{F} \npreceq \mathcal{A F U}$;

- $\mathcal{A O}_{F} \npreceq \mathcal{O}_{F} \mathcal{U}$.

By Proposition 32, it is clear that $\mathcal{A O}_{F} \mathcal{U} \npreceq \mathcal{A U}$.

Next, we show that OPT can be expressed by UNION. We first recall the notion of principal subpattern. Given a pattern $P$, the principal subpattern $\operatorname{ps}(P)$ of $P$ is an $\mathrm{OPT}_{F^{-}}$ free subpattern of $P$ defined as follows (Zhang et al. 2018):

- $\operatorname{ps}((u, v, w))=(u, v, w)$;

- $\operatorname{ps}\left(P_{1}\right.$ UNION $\left.P_{2}\right)=\operatorname{ps}\left(P_{1}\right)$ UNION ps $\left(P_{2}\right)$;

- $\operatorname{ps}\left(P_{1}\right.$ AND $\left.P_{2}\right)=\operatorname{ps}\left(P_{1}\right)$ AND $\operatorname{ps}\left(P_{2}\right)$;

- $\operatorname{ps}\left(P_{1} \mathrm{OPT}_{F} P_{2}\right)=\operatorname{ps}\left(P_{1}\right)$; and

- $\operatorname{ps}\left(P_{1}\right.$ FILTER $\left.F\right)=\operatorname{ps}\left(P_{1}\right)$ FILTER $F$.

Lemma 33. If $P$ is a pattern in $\mathcal{O}_{F} \mathcal{U}$, then $\operatorname{ASK}(P) \equiv_{\mathrm{ASK}}$ $\operatorname{ASK}(\operatorname{ps}(P))$.

By the above lemma, it is easy to see the following result.

Proposition 34. The following hold.

- $\mathcal{O}_{F} \mathcal{U} \preceq \mathcal{U}$;

- $\mathcal{O}_{F} \preceq \mathcal{O}$.

\section{Expressivity of ASK queries with AND}

It is proven that AND can be expressed by OPT and FILTER (Zhang and den Bussche 2014, Proposition 4).

Moreover, we show that both $\mathrm{OPT}_{F}$ and FILTER are necessary for expressing AND. We need a lemma before proving the result.

Lemma 35. Let $P$ be a pattern in $\mathcal{F U}$. If there exists some graph $G$ such that $\llbracket P \rrbracket_{G}$ is nonempty, then there must be a singleton graph $G^{\prime}$ such that $\llbracket P \rrbracket_{G^{\prime}}$ is nonempty.

It is in order to state the main result in this subsection.

Proposition 36. $\mathcal{A} \npreceq \mathcal{F U}$.
By Propositions 34 and 36, $\mathcal{U}$ cannot be expressed by $\mathcal{O}_{F}$ alone.

Corollary 37. $\mathcal{O}_{F} \mathcal{U} \npreceq \mathcal{O}_{F}$.

Based on Proposition 36 and Corollary 37, we can assert that both $\mathrm{OPT}_{F}$ and FILTER are necessary to express AND for ASK queries.

So far, we have investigated the expressivity of ASK queries in all 16 fragments of $\mathcal{A F} \mathcal{O}_{F} \mathcal{U}$ in Figure 3.

\section{Conclusion}

In this paper, we have conducted a systematic study of relative expressivity for various fragments of SPARQL. Besides new results on SELECT queries, we have shown several interesting expressivity results for ASK queries. These results provide a guideline for SPARQL query optimisation and implementation. As a future work, we are going to investigate expressivity of fragments for ASK queries with the presence of some other operators in SPARQL 1.1 such as EXISTS and NOT EXISTS.

\section{Acknowledgments}

This work is supported by the National Key Research and Development Program of China (2017YFC0908401) and the National Natural Science Foundation of China (61972455, 61672377). Xiaowang Zhang is supported by the Peiyang Young Scholars at Tianjin University (2019XRX-0032).

\section{References}

Abiteboul, S.; Hull, R.; and Vianu, V. 1995. Foundations of Databases. Addison-Wesley.

Angles, R., and Gutiérrez, C. 2008a. The expressive power of SPARQL. In Proceedings of th 7th International Semantic Web Conference, ISWC 2008, Karlsruhe, Germany, 114129. Springer.

Angles, R., and Gutiérrez, C. 2008b. Survey of graph database models. ACM Computing Survey 40(1):1:1-1:39.

Angles, R., and Gutiérrez, C. 2010. SQL nested queries in SPARQL. In Proceedings of the 4th Alberto Mendelzon International Workshop on Foundations of Data Management, Buenos Aires, Argentina. CEUR-WS.org.

Angles, R., and Gutiérrez, C. 2016. Negation in SPARQL. In Proceedings of the 10th Alberto Mendelzon International Workshop on Foundations of Data Management, Panama City, Panama. CEUR-WS.org.

Arenas, M., and Pérez, J. 2011. Querying semantic web data with SPARQL. In Proceedings of the 30th ACM SIGMOD Symposium on Principles of Database Systems, PODS 2011, Athens, Greece, 305-316. ACM.

Casanovas, E.; Dellunde, P.; and Jansana, R. 1996. On elementary equivalence for equality-free logic. Notre Dame Journal of Formal Logic 37(3):506-522.

Chandra, A. K., and Harel, D. 1980. Computable queries for relational data bases. Journal of Computing System Science 21(2):156-178. 
Chebotko, A.; Lu, S.; and Fotouhi, F. 2009. Semantics preserving sparql-to-sql translation. Data Knowledge Engineering 68(10):973-1000.

Chekol, M. W. 2016. On the containment of SPARQL queries under entailment regimes. In Proceedings of the 30th AAAI Conference on Artificial Intelligence, Phoenix, Arizona, USA, 936-942. AAAI Press.

Costabello, L.; Villata, S.; and Gandon, F. 2012. Contextaware access control for RDF graph stores. In Proceedings of the 20th European Conference on Artificial Intelligence, ECAI 2012, Montpellier, France, 282-287. IOS Press.

Cyganiak, R.; Wood, D.; and Lanthaler, M. 2014. RDF 1.1 Concepts and Abstract Syntax. W3C Recommendation.

Cyganiak, R. 2005. A relational algebra for SPARQL. Technical report, HP Laboratories Bristol.

Flum, J.; Frick, M.; and Grohe, M. 2002. Query evaluation via tree-decompositions. Journal of ACM 49(6):716-752.

Gottlob, G., and Pieris, A. 2015. Beyond SPARQL under OWL 2 QL entailment regime: Rules to the rescue. In Proceedings of the 24th International Joint Conference on Artificial Intelligence, IJCAI 2015, Buenos Aires, Argentina, 2999-3007. AAAI Press.

Gottlob, G.; Grädel, E.; and Veith, H. 2002. Datalog LITE: a deductive query language with linear time model checking. ACM Transactions Computational Logic 3(1):42-79.

Gottlob, G.; Leone, N.; and Scarcello, F. 2001. The complexity of acyclic conjunctive queries. Journal of ACM 48(3):431-498.

Guido, N. 2015. On the static analysis for SPARQL queries using modal logic. In Proceedings of the 24th International Joint Conference on Artificial Intelligence, IJCAI 2015, Buenos Aires, Argentina, 4367-4368. AAAI Press.

Harris, S., and Seaborne, A. 2013. SPARQL 1.1 Query Language. W3C Recommendation.

Hoffart, J.; Suchanek, F. M.; Berberich, K.; and Weikum, G. 2013. YAGO2: A spatially and temporally enhanced knowledge base from wikipedia. Artificial Intelligence 194:28-61.

Kaminski, M., and Kostylev, E. V. 2016. Beyond welldesigned SPARQL. In Proceedings of the 19th International Conference on Database Theory, ICDT 2016, Bordeaux, France, 5:1-5:18. Schloss Dagstuhl - Leibniz-Zentrum fuer Informatik.

Kaminski, M.; Kostylev, E. V.; and Grau, B. C. 2016. Semantics and expressive power of subqueries and aggregates in SPARQL 1.1. In Proceedings of the 25th International Conference on World Wide Web, WWW 2016, Montreal, Canada, 227-238. ACM.

Kaminski, M.; Kostylev, E. V.; and Grau, B. C. 2017. Query nesting, assignment, and aggregation in SPARQL 1.1. ACM Transactions Database Systems 42(3):17:1-17:46.

Kontchakov, R., and Kostylev, E. V. 2016. On expressibility of non-monotone operators in SPARQL. In Proceedings of the Fifteenth International Conferenceon Principles of Knowledge Representation and Reasoning: , KR 2016, Cape Town, South Africa, 369-379. AAAI Press.
Kostylev, E. V.; Reutter, J. L.; Romero, M.; and Vrgoc, D. 2015. SPARQL with property paths. In Proceedings of the 14th International Semantic Web Conference, ISWC 2015 , Bethlehem, PA, USA, 3-18. Springer.

Kostylev, E. V.; Reutter, J. L.; and Ugarte, M. 2015. CONSTRUCT queries in SPARQL. In 18th International Conference on Database Theory, ICDT 2015, Brussels, Belgium, 212-229. Schloss Dagstuhl - Leibniz-Zentrum fuer Informatik.

Letelier, A.; Pérez, J.; Pichler, R.; and Skritek, S. 2013. Static analysis and optimization of semantic web queries. ACM Transactions on Database Systems 38(4):25:1-25:45.

Nikolaou, C., and Koubarakis, M. 2016. Querying incomplete information in RDF with SPARQL. Artificial Intelligence 237:138-171.

Pérez, J.; Arenas, M.; and Gutiérrez, C. 2009. Semantics and complexity of SPARQL. ACM Transactions on Database Systems 34(3):16:1-16:45.

Pichler, R., and Skritek, S. 2014. Containment and equivalence of well-designed SPARQL. In Proceedings of the 33rd ACM SIGMOD Symposium on Principles of Database Systems, PODS'14, Snowbird, UT, USA, 39-50. ACM.

Polleres, A. 2007. From SPARQL to rules (and back). In Proceedings of the 16th International Conference on World Wide Web, WWW 2007, Banff, Alberta, Canada, 787-796. ACM.

Prud'hommeaux, E., and Seaborne, A. 2008. SPARQL Query Language for RDF. W3C Recommendation.

Schmidt, M.; Meier, M.; and Lausen, G. 2010. Foundations of SPARQL query optimization. In Proceedings of the 13th International Conference, ICDT 2010, Lausanne, Switzerland, 4-33. ACM.

Wood, P. T. 2012. Query languages for graph databases. SIGMOD Record 41(1):50-60.

Zhang, X., and den Bussche, J. V. 2014. On the primitivity of operators in SPARQL. Information Processing Letter 114(9):480-485.

Zhang, X., and den Bussche, J. V. 2015. On the power of SPARQL in expressing navigational queries. The Computer Journal 58(11):2841-2851.

Zhang, X.; den Bussche, J. V.; Wang, K.; and Wang, Z. 2018. On the satisfiability problem of patterns in SPARQL 1.1 . In Proceedings of the 32 AAAI Conference on Artificial Intelligence, (AAAI-18), New Orleans, Louisiana, USA, 20542062. AAAI Press.

Zhang, X.; den Bussche, J. V.; and Picalausa, F. 2016. On the satisfiability problem for SPARQL patterns. Journal Artificial Intelligence Research 56:403-428.

Zhang, X.; Meng, C.; and Zou, L. 2018. Expressivity issues in SPARQL: monotonicity and two-versus threevalued semantics. SCIENCE CHINA Information Sciences 61(12):129102:1-3. 